\title{
Exploring Boltzmann-Factor Distributions of Precipitation-Nuclei in the TEM.
}

Jamie Roberts ${ }^{1}$, P. Fraundorf ${ }^{1,2}$, Jai Kasthuri ${ }^{3}$, and David Osborn ${ }^{2}$.

1. Physics \& Astronomy Department, University of Missouri - St Louis, MO, 63121, USA

2. Center for Nanoscience, University of Missouri - St. Louis, MO, 63121, USA

3. SunEdison Solar Inc., Maryland Heights, MO 63043, USA

Nucleation theory is predicated on evolution of the "precritical cluster distribution", something that in gas and liquid phase nucleation and growth studies is difficult to approach experimentally, except perhaps statistically e.g. via a scattering technique. In solid-state nucleation and growth settings, however, the "quench" often allows one to freeze the distributions in place for a cluster-by-cluster look.

Czochralski semiconductor-silicon is an interesting case in point [1]. Oxygen from the growth crucible, initially uninvited, has proven to be an ally to the device manufacturing process by strengthening the wafer and by precipitating to create defects capable of trapping electrically active impurities, thus improving device yields. There is therefore practical interest, by the integrated-circuit and solar-cell industries, in control of oxygen-cluster behavior. Moreover, unclustered oxygen (in the 10-20 ppm range) may be mapped across a wafer via infrared absorption, while the very earliest stages of oxygen clustering (in the $450-550^{\circ} \mathrm{C}$ range) are connected to "thermal donors" which, albeit avoided by the industry, may be used to characterize clusters via their effects on the bandgap with exquisite sensitivity.

In this note we use oxygen in semiconductor-silicon to discuss: (i) Boltzmann-factor visualizations of sub-critical cluster distributions in the solid-state, and (ii) strategies for exploring them in the TEM.

Figure 1 is a schematic of the Boltzmann probability-factors as a relative measure of the steady-state cluster number/unit-volume, versus cluster size, for (a) the hot silicon after solidification in blue, (b) the "as-grown" distribution in a given part of the ingot after gradual cool-down in the Czochralski puller. This is stepped simply because we lowered the temperature in $50^{\circ} \mathrm{C}$ increments in the simulation, and (c) after one (left) or two (right) "growth steps", the last of these steps at $1000^{\circ} \mathrm{C}$ to facilitate the diffusion needed to precipitate most of the wafer oxygen if post-critical clusters are available.

These schematic distributions evolve simply: Below critical radius tiny clusters grow or evaporate quickly to the Boltzmann steady-state, while above critical radius at the current temperature the distribution simply translates rightward as more oxygen is added. As you can see in Fig. 1, for instance, the $1000^{\circ} \mathrm{C}$ treatment in the absence of an $800^{\circ} \mathrm{C}$ growth step has found too few clusters above critical size at $1000^{\circ} \mathrm{C}$ (the appropriate vertical line) to result in a detectable number of growing precipitates.

Fig. 1 also suggests that as-grown silicon is sure to contain a respectable number of clusters in the verysmall size regime. Heat treatments like that at right (followed by significantly more growth time) is the standard way in which that subcritical distribution is characterized (followed by either TEM or etching), as well as how precipitates are created for use in the device manufacturing process itself.

To be quantitative in finding the number of grown precipitates (or "developed clusters") per unit volume, quantitative ways to measure specimen thickness are needed. In silicon ion-milled specimens, for instance, interstitial $\{111\}$ stacking-faults (created by $\mathrm{Si}$ interstitial displacement during 
precipitation) which intersect specimen top and bottom provide a direct geometric measure. Other methods under development and use for silicon include single-crystal thickness-fringes, energy-filtered TEM, and (at lower spatial-resolution laterally) color-changes of transmitted light [2].

You can see from Fig. 1, we should be able to examine the pre-critical nuclei (or "latent clusters") as their abundance is at least as great. The problem is that these clusters may be un-strained. With neither strain-fields nor a Z-contrast advantage. This means that searches of only very thin regions with minimal surface "noise" will show promise. Hence the quest for specimen-preparation strategies, and instruments able to locate (and characterize) such tiny cluster-distributions in important materials, continues.

\section{References:}

[1] Fumio Shimura (1989) Semiconductor silicon crystal technology (Academic Press, San Diego CA). [2] J. P. McCaffrey and J. Hulse (1998) Micron 29, Issues 2-3, Pages 139-144.
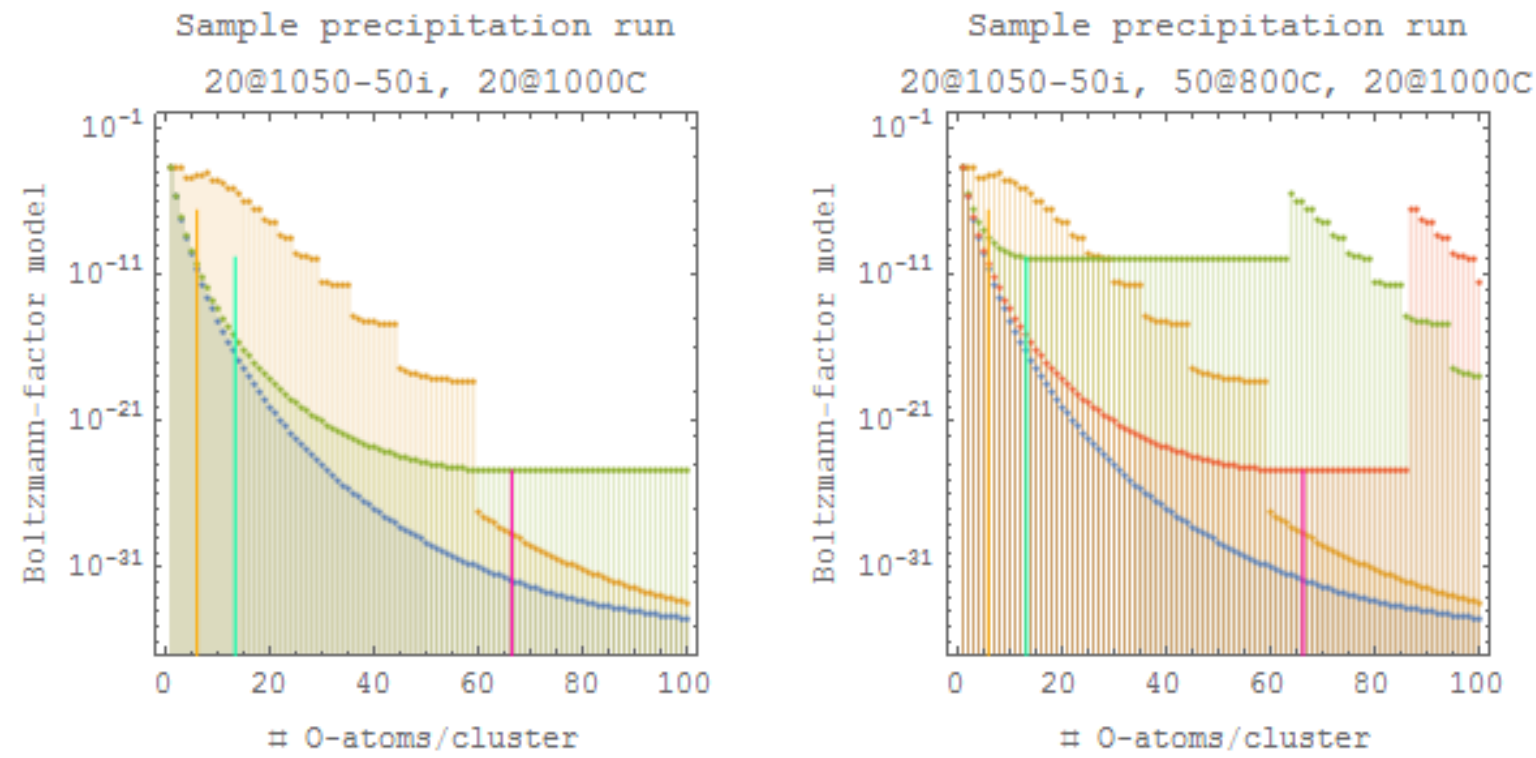

Figure 1. Boltzmann-factor cluster-distributions discussed in the text.

Figure 2. Stacking fault associated with late stage oxygen precipitation, whose known habit plane and intersection with the top and bottom surfaces of the thinned silicon specimen allows for rather precise local thickness determination. The field-width of this $300[\mathrm{keV}]$ electron image is about $3.98[\mu \mathrm{m}]$, making the projected breadth of the stackingfault truncations about 656 [nm]. Multiplying by $\operatorname{Tan}\left[54.7^{\circ}\right]$ for the angle between $\{111\}$ and the $\{100\}$ beam direction gives us a (quite large) local specimen thickness of about 0.92 $[\mu \mathrm{m}]$.

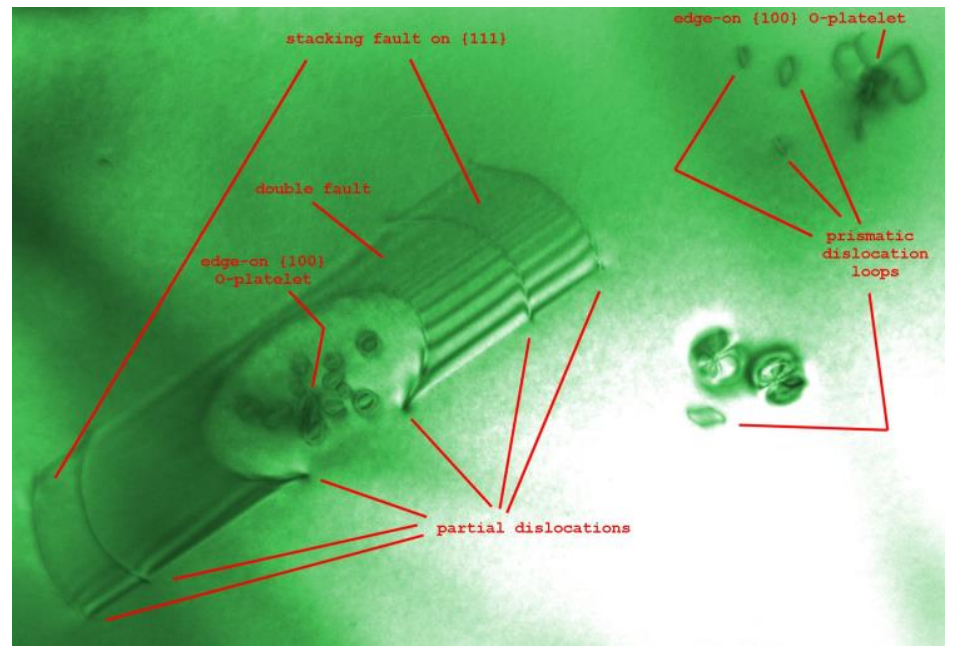

